Bundesgesundheitsbl 2020 $\cdot 63: 729-740$ https://doi.org/10.1007/s00103-020-03148-1 Online publiziert: 25. Mai 2020

(c) Der/die Autor(en) 2020

\author{
Maria Zens ${ }^{1}$ Yvette Shajanian Zarneh ${ }^{2}$ Jürgen Dolle ${ }^{3}$ Freia De Bock ${ }^{2}$ \\ 'Bonn, Deutschland \\ ${ }^{2}$ Bundeszentrale für gesundheitliche Aufklärung, Köln, Deutschland \\ ${ }^{3}$ Lennestadt, Deutschland
}

\section{Digital Public Health - Hebel für Capacity Building in der kommunalen Gesundheitsförderung} Ausgangslage, Entwicklungsfragen,
TEAviisari als modellhafte Implementierung
Digital Public Health (DPH) ist ein sich erst konturierendes neues und umfängliches Feld [1], das u. a. digital unterstützte Public-Health-Maßnahmen und -Programme, digitale Applikationen („Apps“) bis hin zu im Web verfügbaren Gesundheitsinformationen und Social-MediaNutzung umfasst. Weniger Beachtung - zumindest in Deutschland - findet $\mathrm{DPH}$ im Sinne datengetriebener und datenintegrierender Weiterentwicklung von Berichterstattung, Informationssystemen und Evaluation. In diesem Beitrag liegt der Fokus auf der webbasierten, interaktiven Repräsentation von Gesundheitsförderungsdaten, die für Informations-, Steuerungs- und Benchmarkingzwecke genutzt werden können. Das Anliegen dieses Beitrags ist, die Kapazitätsentwicklung kommunaler Gesundheitsförderung mit den integrierenden, analytischen und interaktiven Möglichkeiten digitaler Datenumgebungen zusammenzuführen.

\section{Kommune als Zielpunkt eines Paradigmenwechsels}

In den letzten Jahrzehnten gibt es ein vermehrtes politisches und gesellschaftliches Interesse, Gesundheitsförderung vor allem auf kommunaler Ebene zu implementieren. Meilensteine für diese Entwicklung sind der mit der Otta-
wa-Charta von 1986 für alle PublicHealth-Anstrengungen vollzogene $\mathrm{Pa}$ radigmenwechsel mit seiner expliziten Verpflichtung auf die Stärkung kommunaler Aktivitäten und die Schaffung gesundheitsförderlicher Lebensumstände sowie in Deutschland zuletzt das Präventionsgesetz (2015).

Diese Neuorientierung umfasst in der Verbindung von räumlichem Ansatz [2], intermediärer Ebene [3] und Intersektoralität [4] eine ganze Reihe von strategischen Perspektivänderungen, die auf die Aufwertung von kommunaler Gesundheitsförderung zulaufen. Kommune wird konsequenterweise auch als „Dachsetting“ [5] verstanden. Begriffe wie Verhältnisprävention, Setting, Lebenswelt und Sozialraum zielen auf konkrete multifaktorielle Ermöglichungsstrukturen „vor Ort“ [6], WHOProgramme wie die „Healthy Cities“ [7] setzen solche um.

Der Konsens hierfür ist offenkundig breit: Die „Nationale Präventionskonferenz" (NPK) definiert 2019 die kommunale Ebene als „den wichtigsten Bezugsrahmen für die einzelnen Lebenswelten“ [8]. Die 22 Autorinnen und Autoren des „Memorandums - Prävention und Gesundheitsförderung nachhaltig stärken“ zielen vor allem auf die politikfeldübergreifende Gestaltung ab und messen Kommunen eine wichtige Rol- le zu [9]. Der Spitzenverband der gesetzlichen Krankenversicherung (GKV) mahnt intersektorale Kooperationen an [4] und gibt acht konkrete, auf den kommunalen Kontext bezogene Handlungsempfehlungen [10]. Das Zukunftsforum Public Health sieht trotz dieser zahlreichen Ansatzpunkte noch Verbesserungsbedarf, „insbesondere hinsichtlich Koordinierung, Verbindlichkeit und Transparenz" [11].

Empfehlungen, verstärkt auf ein horizontal über die Sektoren und vertikal über die Politikebenen integriertes Konzept von Gesundheitsförderung hinzuarbeiten, speisen sich nicht zuletzt aus einer gewissen Enttäuschung darüber, dass Projekte insular bleiben und die vielfach und vielerorts gute Präventionspraxis häufig nicht in nachhaltige Strukturen übersetzt werden konnte, wie exemplarisch bei Süß 2013 [12] deutlich wird.

Eine Strategie zur integrierenden Ausgestaltung der präventiven Angebotsstruktur zielt darauf, diese oft kritisierte Inselkultur zu überwinden und „Daten für Taten " sichtbarer und zugänglicher $\mathrm{zu}$ machen. In dieser Situation kann Digital Public Health (DPH) Barrieren überwinden und „Daten für Taten“ sichtbarer und zugänglicher machen. Daten können auf neuartige Weise integriert, strukturiert und disseminiert werden. 
Im Folgenden wird erörtert, warum besonders Capacity Building (CB) auf lokaler und kommunaler Ebene von derartigen technologischen Entwicklungen profitieren kann.

\section{Capacity Building: Entwicklung nachhaltiger Infrastruktur}

Capacity Building wird in der Literatur recht breit aufgefasst. Der Begriff wurde 2006 neu in das WHO Health Promotion Glossary aufgenommen, dort heißt es: „Capacity building is the development of knowledge, skills, commitment, structures, systems and leadership to enable effective health promotion" [13]. Diese Heterogenität $\mathrm{zu}$ strukturieren, ist aus konzeptioneller Sicht eine Herausforderung $[14,15]$. Doch auch wenn der Begriff zunächst unscharf erscheint, fungiert er als Leitidee für engagierte Praxis und ist operativ nützlich. Was davon lässt sich auf den Kontext kommunaler Gesundheitsförderung anwenden und wie kann der praxisorientierte Begriff systematisiert werden?

Die heterogene Literaturlage soll für unseren Kontext auf ein Konzept von $\mathrm{CB}$ enggeführt werden, das auf kommunale Public-Health-Infrastruktur abzielt. Der Systematisierungsvorschlag von Hawe et al. [14] kategorisiert die Literatur in drei verschiedene Grundkonzepte. Das grundständigste ist die Entwicklung von Gesundheitsinfrastruktur und -angeboten („,health infrastructure or service development"), auf einer nächsten Stufe geht es um die Verstetigung („program maintenance and sustainability"), in einer weiteren wird die Fähigkeit benannt, Probleme und Bedarfsstrukturen zu erkennen und angemessene Lösungen zu finden („problem-solving capability of organisations and communities"). Die Kapazitäten, die es zu entwickeln gilt, sind Kompetenzen (Skills), Organisationsstrukturen (Organizational Structures), Ressourcen (Resources) und Verbindlichkeit (Commitment) - also auf Struktur und Organisation bezogene Kategorien, die auf unterschiedliche, thematisch zu bestimmende Handlungsfelder angewendet werden können. Eine weitere Klärung ergibt sich durch Labonte und Laverack [16], die CB zugleich als Mit- tel und Zielpunkt („means“ und „end“) sehen.

Auch wenn CB als Überschrift für Verschiedenes genutzt wird, richten sich die meisten theoretisch ausgerichteten Arbeiten doch auf diese Infrastrukturkomponenten. Exemplarisch kann hier neben den erwähnten auf $[17,18]$ und insbesondere [19] verwiesen werden. Das Ziel des strukturorientierten Ansatzes von CB ist vor allem die Steigerung der Effektivität von Public Health, insofern an der breiten Basis der „health impact pyramid“ [20] angesetzt wird, wo durch dauerhafte Angebotsstrukturen und Multiplikationseffekte effizient und effektiv Einfluss genommen werden kann. In [18] werden 9 Bereiche von CB genannt, die alle organisationsbezogen sind (hier als „domains“ bezeichnet). Aluttis et al. [17] listen 11 Ansätze von CB auf, die sämtlich auf die systemische Ebene (national oder regional) zielen. Hier wird auch ausdrücklich darauf hingewiesen, dass es nicht um die unmittelbare Verbesserung des Gesundheitsstatus gehe, sondern um nachhaltige gute Gesundheitsbedingungen [17]. Labonte und Laverack [16] arbeiten u. a. 2 wesentliche Aspekte heraus: CB ist relational und raumbezogen. $\mathrm{CB}$ als relational zu verstehen ermöglicht bedarfsorientierte Veränderungsprozesse: Welche Kapazitäten wo benötigt werden, kann (und soll) nicht abschließend bestimmt werden; mit dem Raumbezug wird „community capacity“ fokussiert und die konkrete Lage vor Ort. Insofern geht CB immer von vorgefundenen Verhältnissen und vorhandenen Ressourcen aus und kann kein unidirektionaler (Topdown-)Prozess sein. Im zweiten Teil der Publikation [18] betrachten die Autoren die Möglichkeiten von Evaluation und Messung von CB. Hierbei werden vor allem Fragen, die sich beim Ranking von $\mathrm{CB}$ (und dessen Akzeptanz) ergeben, thematisiert. Als Visualisierung wird eine Darstellung verschiedener Bereiche als „spider web“ bevorzugt.

Eine analytische Zusammenschau, die auf der 6th Global Conference on Health Promotion der WHO präsentiert wurde, hebt vor allem auf die Notwendigkeit von Intersektoralität und Mehrebenenhandeln ab. Als Schlüssel für die Effektivität von $\mathrm{CB}$ werden querschnittli- che Ansätze gesehen. Zusammenfassend heißt es, „certain actions are central to effectiveness, such as intersectoral action and interorganizational partnerships at all levels, community engagement and participation in planning and decisionmaking, creating healthy settings $[. .$.$] ,$ political commitment, funding and infrastructure and awareness of the socioenvironmental context" [19].

Festzuhalten ist, dass CB mit Blick auf die Kommune horizontal und vertikal integriert sein muss sowie angebots- und strukturorientiert ist. Die Schaffung public-health-bezogener Infrastruktur wird als Voraussetzung für Health in All Policies (HiAP) verstanden [21]. Capacity ist eine Infrastruktur, die auf Bedarf abgestimmt und als grundständiges Angebot „da ist“, d.h., sie ist auf Dauer angelegt, sollte nicht nur partikular sein und wird nicht maßnahmenbezogen aufgesetzt. Wenn im Folgenden vor allem auf die digitale und informatische Unterstützung von Messung, Monitoring und Vergleich abgehoben wird, soll aber doch betont werden, dass die Zusammenführung und interaktive Nutzbarkeit von CBDaten eine wesentliche Funktion für die sektorenübergreifende intrakommunale Information und Entscheidungsfindung haben - und damit eine Dienstleistung für die Public-Health-Praxis sein sollten. Idealtypisch wären diese unterschiedlichen Funktionen mit denselben strukturierten Datenbeständen (bzw. Teilen davon) $\mathrm{zu}$ realisieren. Nachweise von tatsächlichen Effekten der Implementierung digitalen Monitorings von $\mathrm{CB}$ auf die längerfristige Entwicklung von $\mathrm{CB}$ in den Kommunen stehen unseres Wissens jedoch noch aus.

\section{Herausforderungen für die Operationalisierung und Messung}

Am Beispiel von CB zeigt sich sinnfällig die Dialektik von „pulls of practice“ und „push of research“ [22]. Zum einen geht es dabei um die Verstetigung, Integration und Vernetzung von verteilter Praxis, aber eben auch um das Erkennen und Füllen von Lücken in der vorhandenen Infrastruktur. Voraussetzung für beides ist die Kartierung des Vorhande- 
Bundesgesundheitsbl 2020 • 63:729-740 https://doi.org/10.1007/s00103-020-03148-1

(c) Der/die Autor(en) 2020

\section{Zens · Y. Shajanian Zarneh · J. Dolle · F. De Bock}

\section{Digital Public Health - Hebel für Capacity Building in der kommunalen Gesundheitsförderung. Ausgangslage, Entwicklungsfragen, TEAviisari als modellhafte Implementierung}

\section{Zusammenfassung}

Mit der Ottawa-Charta von 1986 wurde ein Paradigmenwechsel vollzogen, der alle PublicHealth-Anstrengungen auf die Stärkung kommunaler Aktivitäten und die Schaffung gesundheitsförderlicher Lebensumstände orientiert. Ein Schlüssel hierfür ist ",capacity building" (CB), verstanden als die Entwicklung und möglichst nachhaltige Implementierung von strukturellen Kapazitäten - wie koordinierte Datenerhebung, Abstimmungsprozesse über Sektorengrenzen hinweg, dauerhafte Bereitstellung grundständiger Ressourcen in allen Bereichen der Gesundheitsförderung "vor Ort".

Vielfältige Anstrengungen und dreieinhalb Jahrzehnte später sehen wir uns noch viel zu oft Infrastrukturdefiziten, fragmentierten Public-Health-Landschaften und Hemmnissen für die intersektorale Zusammenarbeit ge- genüber. Während der theoretische Konsens über das Notwendige breit erscheint, bleibt es eine Herausforderung, diese theoretischen Einsichten in die Praxis umzusetzen. In dieser Situation kann Digital Public Health (DPH) dazu beitragen, Barrieren zu überwinden und „Daten für Taten" sichtbarer und zugänglicher zu machen. Durch DPH können Daten auf neuartige Weise integriert, strukturiert und disseminiert werden.

In diesem Beitrag wird erörtert, wie die Kapazitätsentwicklung auf lokaler und kommunaler Ebene von technologischen Entwicklungen profitieren und was DPH für die Bereitstellung von Informationsservices im Bereich Public Health Capacity tun kann. Der Fokus liegt auf der webbasierten, interaktiven Repräsentation von Gesundheitsförderungsdaten, die für Informations-, Steuerungs- und Benchmarkingzwecke genutzt werden können. Als Beispiel aus der Public-HealthPraxis wird das Tool "TEAviisari" (National Institute for Health and Welfare, Finnland) vorgestellt.

Die EU-Ratspräsidentschaft Deutschlands 2020 - u. a. mit den Themen Digitalisierung und gemeinsamer Gesundheitsdatenraum - bietet die Gelegenheit, die Kapazitätsentwicklung in der kommunalen Gesundheitsförderung auch hierzulande entscheidend voranzubringen.

Schlüsselwörter

Digitalisierung · Kommunale Gesundheit . Informationssysteme · Datenvisualisierung . Partizipation

\section{Digital public health—leverage for community capacity building in health promotion. Current situation, developmental issues and TEAviisari as a model implementation}

\section{Abstract}

In 1986, the Ottawa charter marked a paradigm shift for public health, putting the focus on strengthening community action and on creating supportive environments for health. A key to this is "capacity building" (CB), which we understand as the development and sustainable implementation of structural capacities, e.g. coordinated data collection, collaboration processes across sectors and reliable provision of basic resources in all areas of local health promotion.

Many efforts and three and a half decades later we still envisage infrastructure deficits, scattered public health landscapes and restraints to intersectoral cooperation much too often. While agreement on the theoretical insights on what is needed appears to be broad, translating these insights into practice remains a challenge. In this situation, digital public health (DPH) can contribute to overcoming barriers and making knowledge for action more visible and more accessible. With DPH, data can be integrated, structured and disseminated in novel ways.

We discuss why $C B$ at the local level could benefit from technological advances and what DPH might do for the provision of information services on public health capacity. Our focus is on the web-based, interactive representation of public health data for use in information, governance or benchmarking processes. As an example from public health practice, the Finnish tool TEAviisari (National Institute for Health and Welfare, Finland) is presented. The 2020 EU Council Presidency of Germany - with the topics of digitalisation and the common European health data space - offers opportunities to decisively advance the development of $\mathrm{CB}$ in health promotion in this country.

\section{Keywords}

Digitalization · Community health · Information systems · Data visualisation Participation nen nach einem möglichst konsensuellen und nachvollziehbaren Schema.

Dass Kapazitäten gemessen werden sollen, ist wohl kaum strittig; aber gerade wenn es um Vergleichbarkeit und auch Gerechtigkeit in der Ressourcenverteilung geht, muss es einen Diskurs geben, wie und was gemessen werden soll und auch wie Neues integriert und messbar gemacht werden kann [23].
Was sind geeignete Maßzahlen für die organisationsbezogenen Kapazitäten, wie werden sie auf Domänen angewendet, wie werden geeignete Daten erhoben, vorhandene Daten integriert und auf die kommunale Ebene bezogen (aggregiert oder gesplittet), wie kann ein landesweites Framework aussehen, das relevante Informationen für Vergleich und Steuerung bietet? Jeder dieser Punkte ist herausfordernd. Das Anliegen, vorhan- dene Daten zu mappen, ist nicht nur mit unterschiedlichen Datenbeständen [24, 25] konfrontiert, sondern grundsätzlicher mit einem Terminologieproblem [23].

Deutschland hat bei der Beantwortung solcher Fragen noch vieles vor sich und liegt im internationalen Vergleich bei der Nutzung von Daten für die Public-Health-Forschung insgesamt eher zurück [26]. Ein wesentlicher Schritt zur 
verbesserten Nutzung wäre die Integration von - bis hinunter auf Kommunalebene - zunächst getrennt erhobenen Daten, wie Stockmann et al. [27] in einer empirischen Zusammenstellung deutlich machen. Wenn Burgun et al. [26] aber feststellen: „Research in public health is transitioning from siloed systems to more accessible and re-usable data resources", liegen hierin neue Chancen der Datenverknüpfung, die wesentlich mit der Implementierung digitaler Systeme und der Nutzung informatischer Expertise verbunden ist. Das gilt gleichermaßen für ganz unterschiedliche Daten; CB könnte aber besonders profitieren, zum einen, weil die Datenlage divers ist und die Zuständigkeiten für relevante Daten in Kommunen weit verteilt sind, und auch, weil es in diesem Bereich viele gute Praxisbeispiele, Pilot- und Einzelprojekte gibt. Für diese sind Vergleichbarkeit und Datenintegration über die Einzelerhebung hinaus zunächst meist nachrangig - im Gegensatz beispielsweise zur kommunalen Gesundheitsberichterstattung (GBE), für die das längst Ziel ist. Es liegt nahe, diese und weitere gut aufgestellte kommunale Datenpools als inhaltlich und technisch anschlussfähige Informationsangebote $\mathrm{zu}$ sehen, die perspektivisch Teil von CBInformationssystemen werden könnten.

Beider Messung kommunaler Kapazitäten geht es um konkrete organisationsbezogene Maßzahlen für Public-HealthDomänen. Um zu einer validen Datenbasis zu gelangen, bedarf es eines geeigneten Frameworks - gegebenenfalls mit modularen, inkrementell aufzubauenden Elementen. Zugleich sollten beide "Achsen“- Strukturerfassung (die strukturellen „capacities") und Domänenbestimmung (z. B. Ernährung, Bewegung, Angebote für alte Menschen, Suchtprävention) - offen für Erweiterungen und Veränderungen sein. Aus der Perspektive der abstrakten Modellierung - oder auch in politischen Top-down-Prozessen - mögen solche Ergänzungen selbstverständlich erscheinen, in der multisektoralen Praxis von Public Health ist es aber durchaus nicht immer einfach und angesichts der zahlreichen beteiligten Stakeholder auch nicht einfach zu konsentieren.
In praktischen Beispielen der Kapazitätsmessung spielen die beiden „Achsen" ineinander und werden häufig zu Profilen unterschiedlicher Aggregationsstufen, zu Domänenansichten o. Ä. aufbereitet, womit sie unmittelbar anschaulich und nutzbar werden, wie weiter unten gezeigt werden wird. Innovative Datenvisualisierungen und die digitalen Möglichkeiten, kuratierte Datenbestände multipel einzusetzen, spielen hierbei eine zentrale Rolle. Wie genau die thematischen Bereiche zustande kommen, was warum ausgewählt wurde oder ob nicht auch andere Informationen sinnvoll $\mathrm{zu}$ integrieren wären, wird nicht immer expliziert und folgt wohl auch pragmatischen Imperativen (vorhandene Daten, Vorgängerprojekte etc.). Das ist aus wissenschaftlicher Sicht als Limitation anzusehen, schmälert aber nicht den praktischen Wert, den einzelne Angebote für genau den Bereich haben können, den sie abdecken.

Eine entscheidende Frage ist, ob und wie vergleichende Betrachtungen und Analysen vorgenommen werden. Es würde den Rahmen sprengen, hier auf die damit verbundenen Chancen und Probleme einzugehen. Die niederländische Publikation Dare to compare [28] zu den Europäischen Gesundheitsindikatoren (ECHI) zeigt „apples and oranges" als Umschlagabbildung und illustriert damit das Grundproblem der Vergleichbarkeit sinnfällig. Was ist auf kommunaler Ebene sinnvoll? Ein einfaches Maß scheint der Vergleich mit dem Durchschnitt zu sein, aber auch hier ist die Bestimmung der Bezugsgröße nicht trivial (Durchschnitt wovon? Von allen Kommunen, Kommunen ähnlicher Größe, Kommunen mit ähnlichen finanziellen Möglichkeiten, Kommunen mit ähnlicher soziodemografischer Struktur? Wie kann Ähnlichkeit - „likenes““ - definiert und gemessen werden?). Genau zu bewerten ist auch, was Benchmark, Rating oder Ranking leisten (sollen) und wo sie nutzbringend eingesetzt werden können. Und wieweit sind der Konkurrenzökonomie entlehnte Begriffe und Verfahren überhaupt hilfreich, wenn möglichst flächendeckend möglichst gute gesundheitsförderliche Verhältnisse geschaffen werden sollen? Die partizipatorische, motivierende und faire Gestaltung gerade von Bewertungsinstrumenten ist wichtig - z.B. für die Förderung von gleichen Gesundheitschancen und die Allokation von Ressourcen.

Das ist Voraussetzung für die Akzep$\tan z$ von Vergleichen und einen funktionierenden ,data action cycle "im Bereich der Kapazitätsentwicklung kommunaler Gesundheitsförderung, bei dem „Daten für Taten“ genutzt werden sollen.

\section{Digital Public Health als Hebel}

Für eine landesweite Kapazitätsentwicklung kommunaler Gesundheitsförderung ist eine valide Datengrundlage - idealtypisch eine Vollerhebung mit einem standardisierten Set von Kernindikatoren, das in ein strategisches Rahmenwerk eingebettet ist - essenziell; es dürfte auch Konsens sein, dass es eine solche in Deutschland derzeit noch nicht gibt und die Integration vorhandener Informationen und Datenbestände vor großen Hindernissen steht. In dieser Ausgangslage ist es naheliegend, nach Mitteln Ausschau zu halten, diese Hindernisse zu umgehen. Mit Daten im Mittelpunkt bietet Digital Public Health solche Möglichkeiten.

Defizite in anderen Bereichen können durch digitale Entwicklungen nicht wettgemacht werden; solche Entwicklungen können aber zu Verbesserungen in einer ganzen Reihe relevanter Punkte beitragen. Gesundheit ist ein wichtiges Feld für „data-driven innovation“ (DDI) - für datengetriebene Innovation, im gleichnamigen Report der Organisation für wirtschaftliche Zusammenarbeit und Entwicklung (OECD) von 2015 wird ihr ein eigenes Kapitel gewidmet [29]. Unter anderem wird hier auf die Bedeutung für „understanding and managing population and public health" [29] hingewiesen.

DPH eröffnet in den Bereichen Datenerhebung, Datenintegration, Datenverfügbarkeit neue Möglichkeiten und kann als Hebel funktionieren, um bislang Vernachlässigtes oder Unverbundenes umzusetzen, zu integrieren und zu beschleunigen. Eine zentrale Rolle spielt dabei die Entwicklung der Public Health Informatics, eines Felds, das wie Dixon 
et al. [30] feststellen, beständig an Breite, Tiefe und Komplexität zunimmt. Khoury et al. sehen einen tief greifenden datenbezogenen Wandel ,in epidemiology, the fundamental science of public health, to include and integrate disparate sources of data" [31].

Datenerhebung. Eine Infrastruktur für die Erhebung von Daten zur kommunalen Gesundheitsförderung, die auf digitale Technologien setzt, kann auf diese Weise einfacher, kostengünstiger und schneller agieren. Webbasierte Erhebungen bieten zudem erweiterte Möglichkeiten der Begleitung, Erinnerung und Incentivierung. Insgesamt können partizipatorische Ansprüche hier unmittelbarer umgesetzt werden, z. B. durch Kontextinformationen, Subskription der Erhebungsergebnisse, Feedbackfunktionen u.v.m. Bei verpflichtenden Datenlieferungen sind geringe Nonresponse-Raten zu erwarten. Vergleichende Untersuchungen zu Online- und traditionellen Surveys erreichen aber auch bei randomisierten Stichproben gute Responseraten für die digitale Befragung [32].

Datenintegration. Durch computergestützte Verfahren lassen sich sorgfältig erhobene und kuratierte Datenbestände über Metadaten verknüpfen. Daten zu Gesundheitsförderung oder Gesundheitsstatus können über geografische Attribute aggregiert oder gesplittet werden, sofern diese Attribute in hinreichender Granularität und Präzision vorliegen. Relevante Daten aus anderen Informationsbereichen können genutzt werden (z.B. Informationen zur sozioökonomischen Lage, zum Gesundheitsverhalten, zur Primärversorgung oder zur Verfügbarkeit von Sportstätten in einer Kommune, eventuell auch Routinedaten). Für Datenintegration mit kommunalem oder regionalem Bezug sind Entwicklungen im Bereich geografischer Informationssysteme (GIS) relevant [33, 34].

Datenverfügbarkeit. Digitale Technologien beschleunigen Erhebungsprozesse und erleichtern es, aus den Daten gewonnene Informationen zeitnah und einfach zugänglich zu machen. Digitalität er- möglicht die ubiquitäre Verfügbarkeit von Information. Von der webbasierten Dissemination von Public-Health-Daten können unterschiedliche Benutzergruppen (Forschende, Entscheiderinnen, Praktiker, interessierte Allgemeinheit) profitieren. Ein Vorzug digitaler Wissensrepräsentation ist dabei, dass für verschiedene Informationsbedürfnisse unterschiedliche Ansichten auf dasselbe erzeugt werden.

Die Verpflichtung aufFAIR Data Principles ist ein wesentlicher Entwicklungsschritt, der das unterstützt. Mit FAIR sind die Eigenschaften „auffindbar“ („findable“), „zugänglich“ („accessible“), ,interoperabel“ (,interoperable“) und „wiederverwendbar“ („re-usable“) gemeint [35]. Zum einen zielt das auf die wissenschaftliche Qualität der Daten ab, ihre Transparenz, Verfügbarkeit und Reproduzierbarkeit. Ebenso wichtig (und neu) ist der Ansatz für die Ermöglichung computergestützter, algorithmischer Datennutzung, maschineller Datensuche und -verknüpfung und damit datengetriebener Innovation. FAIR bezieht sich grundsätzlich auf alle Arten von Forschungsdaten. Bei den Daten, die für CB genutzt werden könnten, handelt es sich größtenteils um bereits erfasste Daten (bottom up), die selbst nicht genuin digital sein müssen, aber computergestützt zusammengeführt und durch Visualisierung analytisch aufbereitet werden sollten, um so beispielsweise die datenbasierte Steuerung von Maßnahmen zu unterstützen. Bei Berücksichtigung genuin digitaler Daten müssten die weiteren insbesondere auch die unintendierten Implikationen von Digitalisierung in den Blick genommen werden [36]. Mit Blick auf CB-Daten sind vor allem Interoperabilität, die Verknüpfung von Daten (z. B. über „linked open data“) und die Unterstützung von Entscheidungsprozessen wichtig. Daher sind die von Burgun et al. [26] gelisteten 5 Bereiche, in denen die FAIR-Prinzipien positive Effekte spezifisch für Public Health erzeugen, auch für CB gültig: „data discovery“, „[access] to aggregated data“, „, [access] to individual data“, „services for hypothesis-driven studies through models and algorithms“, „services for knowledge discovery with big data approaches“ [26].
All dies weist in Richtung integrierter Frameworks und interoperabler Daten, die Zersplitterung, Sektoralisierung, „Insel“- und „Silo“-Effekte zu überwinden helfen und aufverschiedenen Ebenen nutzbar sind. In die zukünftige Erhebung von Gesundheits- und Gesundheitsförderungsdaten sollten diese Überlegungen einfließen. Für die europäische Ebene zeigt Carinci [37], wie eine solche Infrastruktur aussehen könnte; die Anforderungen von Multidisziplinarität, effizienter Dissemination und Einbindung verschiedener Benutzergruppen lassen sich auch auf die kommunale Ebene übertragen.

Soll das Digitale in Public Health mehr leisten, als den elektronischen Download von analog Bekanntem zu ermöglichen, lohnt es, den Bereich der analytischen Datenvisualisierung [38, 39] verstärkt anzugehen. „Intelligente“ Visualisierungstechniken sind grundsätzlich gefordert, weil nichtlineare Darstellungsweisen (z. B. benutzerzentrierte Dashboards) und interaktive Zugriffe als Mehrwert digitaler Informationsaufbereitung in zugängliche Benutzeroberflächen umgesetzt werden müssen (benutzerorientierte Gestaltung). Das gilt besonders, wenn es um interpretierende und bewertende Repräsentationen wie Ranking, Rating, Benchmark und Vergleich geht oder darum, eine Fülle von Informationen auf einen Blick verfügbar zu machen.

Gamache et al. [40] nennen in ihrer Analyse u.a. neue Visualisierungstechniken als wesentlichen Trend der Public Health Informatics. Zakkar und Sedig [41] sowie Manorat et al. [42] verweisen auf die Leistung solcher Techniken für Steuerung und professionelle Entscheidungsfindung in verschiedenen Bereichen, grundsätzlicher noch Sedig et al. [43]. Um komplexe Prozesse der Problemerkennung und vorausschauenden Einschätzung, bedarfsgerechten und effizienten Planung unterstützen zu können, müssen solche Systeme strukturiert auf domänenspezifischem Wissen aufsetzen und möglichst intuitiv zugänglich sein. Insbesondere die interaktive analytische Datenvisualisierung kann eine neuartige Arbeitsumgebung bieten und ist eine genuine Leistung digitaler Technologie 


\section{Leitthema}

Tab. 1 Übersicht zu exemplarischen DPH-Angeboten mit kommunalen Public-Health-Daten, Vergleichs- oder Benchmarkfunktion sowie interaktiven Visualisierungen

\begin{tabular}{|c|c|c|c|c|c|}
\hline Name & $\begin{array}{l}\text { San Francisco } \\
\text { City Performance } \\
\text { Scorecards } \\
\text { Public Health Bench- } \\
\text { marking }\end{array}$ & $\begin{array}{l}\text { Kansas Health Matters } \\
\text { Community Health Dashboards }\end{array}$ & $\begin{array}{l}\text { Public Health } \\
\text { England } \\
\text { Public Health } \\
\text { Dashboard }\end{array}$ & SCOTPHO Profiles & TEAviisari \\
\hline URL & $\begin{array}{l}\text { https://sfgov. } \\
\text { org/scorecards/ } \\
\text { benchmarking/ } \\
\text { public-health } \\
\text { https://sfgov.org/ } \\
\text { scorecards/public- } \\
\text { health/public-health } \\
\text { (Zugriff 04.11.2019) }\end{array}$ & $\begin{array}{l}\text { https://www. } \\
\text { kansashealthmatters.org/ } \\
\text { indicators } \\
\text { https://kchealthmatters.org/ } \\
\text { community-dashboard } \\
\text { (Zugriff 04.11.2019) }\end{array}$ & $\begin{array}{l}\text { https://fingertips. } \\
\text { phe.org.uk/public- } \\
\text { health-dashboard- } \\
\text { ft\#page/0/gid/ } \\
\text { 1938133143/pat/6/ } \\
\text { par/E12000009/ati/ } \\
\text { 102/are/E06000022 } \\
\text { (Zugriff 04.11.2019) }\end{array}$ & $\begin{array}{l}\text { https://scotland. } \\
\text { shinyapps.io/ } \\
\text { ScotPHO_profiles_ } \\
\text { tool/ } \\
\text { (Zugriff 04.11.2019) }\end{array}$ & $\begin{array}{l}\text { https://teaviisari.fi/ } \\
\text { teaviisari/en/index? } \\
\text { (Zugriff 04.11.2019) }\end{array}$ \\
\hline $\begin{array}{l}\text { Betreiber, } \\
\text { Land }\end{array}$ & $\begin{array}{l}\text { City and County of } \\
\text { San Francisco, U.S.A. }\end{array}$ & $\begin{array}{l}\text { Kansas Health Matters Partner- } \\
\text { ship, Kansas, U.S.A. }\end{array}$ & $\begin{array}{l}\text { Public Health Eng- } \\
\text { land (Regierungs- } \\
\text { behörde des briti- } \\
\text { schen Ministeriums } \\
\text { für Gesundheit und } \\
\text { Soziales), England }\end{array}$ & $\begin{array}{l}\text { The Scottish Public } \\
\text { Health Observatory } \\
\text { (ScotPHO), Leitung: } \\
\text { ISD Scotland, NHS } \\
\text { Health Scotland, } \\
\text { Schottland }\end{array}$ & $\begin{array}{l}\text { National Institute for } \\
\text { Health and Welfare } \\
\text { (THL), Finnland }\end{array}$ \\
\hline Kernaussage & $\begin{array}{l}\text { "City Performance } \\
\text { Scorecards provide } \\
\text { timely information } \\
\text { on the efficiency and } \\
\text { effectiveness of San } \\
\text { Francisco government } \\
\text { in eight highlighted } \\
\text { service areas." }\end{array}$ & $\begin{array}{l}\text { "Learn about your community's } \\
\text { health and wellness." }\end{array}$ & $\begin{array}{l}\text { "Increasing the } \\
\text { transparency of } \\
\text { public health data." } \\
\text { "See how your } \\
\text { local authority } \\
\text { compares." }\end{array}$ & $\begin{array}{l}\text { "Working to improve } \\
\text { Scotland's health } \\
\text { and reduce health } \\
\text { inequalities." }\end{array}$ & $\begin{array}{l}\text { "TEAviisari shows the } \\
\text { direction of health } \\
\text { promotion capacity } \\
\text { building in munici- } \\
\text { palities. Search and } \\
\text { compare results." }\end{array}$ \\
\hline Ziel & $\begin{array}{l}\text { "[Benchmarking] re- } \\
\text { sults provide useful } \\
\text { context for the pub- } \\
\text { lic and policymakers } \\
\text { to assess how San } \\
\text { Francisco compa- } \\
\text { res to similar peer } \\
\text { jurisdictions and to } \\
\text { identify areas for fur- } \\
\text { ther research and } \\
\text { awareness." }\end{array}$ & $\begin{array}{l}\text { "The Kansas Health Matters web- } \\
\text { site brings community health- } \\
\text { related statistical data, local re- } \\
\text { sources and a wealth of infor- } \\
\text { mation to one, accessible, user- } \\
\text { friendly location. The intent is to } \\
\text { give our communities the tools } \\
\text { they need to read and understand } \\
\text { the public health indicators that } \\
\text { affect the quality of our residents' } \\
\text { lives. The tools help the com- } \\
\text { munity set goals and evaluate } \\
\text { progress." }\end{array}$ & $\begin{array}{l}\text { "The purpose of } \\
\text { the dashboard is } \\
\text { to support local } \\
\text { decision-making by } \\
\text { bringing existing } \\
\text { comparative data } \\
\text { into one place and } \\
\text { making it accessible } \\
\text { and meaningful to } \\
\text { a wide audience." }\end{array}$ & $\begin{array}{l}\text { „The ScotPHO website } \\
\text { is designed to be use- } \\
\text { ful to those carrying } \\
\text { out population needs } \\
\text { assessment. } \\
\text { It should also be use- } \\
\text { ful for those making } \\
\text { decisions about priori- } \\
\text { tisation, and for those } \\
\text { planning services." }\end{array}$ & $\begin{array}{l}\text { „TEAviisari describes } \\
\text { municipalities' activi- } \\
\text { ties to promote their } \\
\text { residents' health. } \\
\text { TEAviisari is designed } \\
\text { to support munici- } \\
\text { palities, regions and } \\
\text { schools in the plan- } \\
\text { ning and management } \\
\text { of health promotion } \\
\text { work." }\end{array}$ \\
\hline $\begin{array}{l}\text { Status, Lauf- } \\
\text { zeit }\end{array}$ & Laufend, seit 2016 & Laufend, seit ca. $2012^{\mathrm{a}}$ & Laufend, seit 2017 & Laufend, seit 2018 & Laufend, seit 2010 \\
\hline Zugang & Open Access & Open Access & Open Access & Open Access & Open Access \\
\hline Ebene & $\begin{array}{l}\text { Kommune, Bundes- } \\
\text { staat, landesweit }\end{array}$ & $\begin{array}{l}\text { Kommune, Bundesstaat, landes- } \\
\text { weit }\end{array}$ & $\begin{array}{l}\text { Kommune, Region, } \\
\text { landesweit }\end{array}$ & $\begin{array}{l}\text { Kommune, Region, } \\
\text { landesweit }\end{array}$ & $\begin{array}{l}\text { Kommune, Region, } \\
\text { landesweit }\end{array}$ \\
\hline \multirow[t]{2}{*}{$\begin{array}{l}\text { Themen- } \\
\text { bereich }\end{array}$} & $\begin{array}{l}\text { Public Health (sowie } 7 \\
\text { weitere Bereiche) }\end{array}$ & $\begin{array}{l}\text { Gesundheit, Demografie, Un- } \\
\text { gleichheit }\end{array}$ & Public Health & Public Health & $\begin{array}{l}\text { Health Promotion } \\
\text { Capacity Building }\end{array}$ \\
\hline & $\begin{array}{l}\text { Spezifische CB-Daten: } \\
\text { nein }^{\mathrm{b}}\end{array}$ & Spezifische CB-Daten: nein ${ }^{b}$ & $\begin{array}{l}\text { Spezifische CB- } \\
\text { Daten: } \text { nein }^{b}\end{array}$ & $\begin{array}{l}\text { Spezifische CB-Daten: } \\
\text { nein }^{\mathrm{b}}\end{array}$ & $\begin{array}{l}\text { Spezifische CB-Daten: } \\
\text { ja, } 7 \text { "Dimensionen“: } \\
\text { Commitment, Ma- } \\
\text { nagement, Monitoring } \\
\text { and Needs Assess- } \\
\text { ment, Resources, } \\
\text { Common Practices, } \\
\text { Participation sowie } \\
\text { domänenspezifische } \\
\text { Maße }\end{array}$ \\
\hline
\end{tabular}




\begin{tabular}{|c|c|c|c|c|c|}
\hline Name & $\begin{array}{l}\text { San Francisco } \\
\text { City Performance } \\
\text { Scorecards } \\
\text { Public Health Bench- } \\
\text { marking }\end{array}$ & $\begin{array}{l}\text { Kansas Health Matters } \\
\text { Community Health Dashboards }\end{array}$ & $\begin{array}{l}\text { Public Health } \\
\text { England } \\
\text { Public Health } \\
\text { Dashboard }\end{array}$ & SCOTPHO Profiles & TEAviisari \\
\hline Domänen & $\begin{array}{l}\text { Public Health: Health } \\
\text { Landscape, Health } \\
\text { Outcomes, Health } \\
\text { Spending }\end{array}$ & $\begin{array}{l}\text { Economy, Education, Environ- } \\
\text { ment, Government \& Politics, } \\
\text { Public Safety, Social Environment, } \\
\text { Transportation }\end{array}$ & $\begin{array}{l}\text { Best Start in Life, } \\
\text { Child Obesity, Drug } \\
\text { Treatment, Alcohol } \\
\text { Treatment, Tobacco } \\
\text { Control, NHS Health } \\
\text { Checks, Sexual } \\
\text { and Reproductive } \\
\text { Health, Air Quality }\end{array}$ & $\begin{array}{l}\text { Behaviours, Social } \\
\text { Care \& Housing, En- } \\
\text { vironment, Life Ex- } \\
\text { pectancy \& Mortality, } \\
\text { Women's \& Children's } \\
\text { Health, Immunisa- } \\
\text { tions \& Screening, } \\
\text { Economy, Crime, Men- } \\
\text { tal Health, III Health \& } \\
\text { Injury, Education }\end{array}$ & $\begin{array}{l}\text { Primary Health Care, } \\
\text { Physical Activity, Mu- } \\
\text { nicipal Management, } \\
\text { Vocational Educa- } \\
\text { tion, Upper Secondary } \\
\text { Education, Basic Edu- } \\
\text { cation, Culture }\end{array}$ \\
\hline Indikatoren & $\mathrm{Ja}$ & $\mathrm{Ja}$ & Ja & Ja & Ja \\
\hline $\begin{array}{l}\text { Datentranspa- } \\
\text { renz, } \\
\text { Dateninte- } \\
\text { gration, } \\
\text { Fortschrei- } \\
\text { bung }\end{array}$ & $\begin{array}{l}\text { Ja } \\
\text { Verschiedene Daten- } \\
\text { quellen } \\
\text { Kontinuierliche Up- } \\
\text { dates }\end{array}$ & $\begin{array}{l}\text { Ja } \\
\text { Verschiedene Datenquellen } \\
\text { Regelmäßige Updates }\end{array}$ & $\begin{array}{l}\text { Ja } \\
\text { Verschiedene Da- } \\
\text { tenquellen } \\
\text { Regelmäßige Up- } \\
\text { dates }\end{array}$ & $\begin{array}{l}\text { Ja } \\
\text { Verschiedene Daten- } \\
\text { quellen } \\
\text { Regelmäßige Updates }\end{array}$ & $\begin{array}{l}\text { Ja } \\
\text { Verschiedene Daten- } \\
\text { quellen } \\
\text { Regelmäßige Updates }\end{array}$ \\
\hline $\begin{array}{l}\text { Vergleich } \\
\text { Bewertung }\end{array}$ & $\begin{array}{l}\text { Ja } \\
\text { Benchmarking, Ran- } \\
\text { king, Rating }\end{array}$ & $\begin{array}{l}\text { Ja } \\
\text { Benchmarking, Rating }\end{array}$ & $\begin{array}{l}\text { Ja } \\
\text { Benchmarking, } \\
\text { Rating, Ranking }\end{array}$ & $\begin{array}{l}\text { Ja } \\
\text { Benchmarking, Rat- } \\
\text { ing, Ranking }\end{array}$ & $\begin{array}{l}\text { Ja } \\
\text { Benchmarking, Rating, } \\
\text { Ranking }\end{array}$ \\
\hline Visualisierung & $\begin{array}{l}\text { Dashboard, Diagram- } \\
\text { me, Trend, Target, } \\
\text { Ampelfarben }\end{array}$ & $\begin{array}{l}\text { Dashboard, Karte, Diagramm, } \\
\text { Tabelle, Target, Gradmesser, Am- } \\
\text { pelfarben }\end{array}$ & $\begin{array}{l}\text { Dashboard, Karte, } \\
\text { Diagramm, Tabelle, } \\
\text { Trend, Ampelfarben }\end{array}$ & $\begin{array}{l}\text { Dashboard, Trend, } \\
\text { Spine, Snapshot, } \\
\text { Karte, Ampelfarben }\end{array}$ & $\begin{array}{l}\text { Dashboard, Gradmes- } \\
\text { ser, Diagramm, Karte, } \\
\text { Trend, Ampelfarben }\end{array}$ \\
\hline $\begin{array}{l}\text { Interaktive } \\
\text { Abfragen }\end{array}$ & $\mathrm{Ja}$ & $\mathrm{Ja}$ & $\mathrm{Ja}$ & $\mathrm{Ja}$ & Ja \\
\hline $\begin{array}{l}\text { Integration } \\
\text { mit weiteren } \\
\text { Angeboten }\end{array}$ & $\mathrm{Ja}$ & $\mathrm{Ja}$ & Ja & $\mathrm{Ja}$ & Ja \\
\hline $\begin{array}{l}\text { Benutzer- } \\
\text { unter- } \\
\text { stützung }\end{array}$ & $\begin{array}{l}\text { Hintergrund- und } \\
\text { Kontextinformationen } \\
\text { per Klick/Mouse over, } \\
\text { Hintergrundinforma- } \\
\text { tionen auf Da- } \\
\text { taSF (https://data. } \\
\text { sfgov.org/City- } \\
\text { Management-and- } \\
\text { Ethics/Scorecard- } \\
\text { Measures/kc49-udxn) } \\
\text { (Zugriff 04.11.2019) }\end{array}$ & $\begin{array}{l}\text { FAQ, Hintergrund- und Kontext- } \\
\text { informationen per Klick/Mouse } \\
\text { over, zahlreiche Zusatzinformatio- } \\
\text { nen }\end{array}$ & $\begin{array}{l}\text { Technical Guides, } \\
\text { Hintergrund- und } \\
\text { Kontextinforma- } \\
\text { tionen per Klick/ } \\
\text { Mouse over, detail- } \\
\text { lierte Informationen } \\
\text { über „Definitions" }\end{array}$ & $\begin{array}{l}\text { Tour of the Tool, Quick } \\
\text { Reference Guide, de- } \\
\text { taillierte Informatio- } \\
\text { nen über "Definitions" }\end{array}$ & $\begin{array}{l}\text { Manual, Hintergrund- } \\
\text { und Kontextinforma- } \\
\text { tionen per Klick/Mouse } \\
\text { over/Infobutton, mehr- } \\
\text { sprachig }\end{array}$ \\
\hline $\begin{array}{l}\text { Download } \\
\text { (von Daten, } \\
\text { Grafiken, } \\
\text { Tabellen) }\end{array}$ & $\begin{array}{l}\text { Daten über Da- } \\
\text { taSF (https://data. } \\
\text { sfgov.org/City- } \\
\text { Management-and- } \\
\text { Ethics/Scorecard- } \\
\text { Measures/kc49-udxn) } \\
\text { (Zugriff 04.11.2019) }\end{array}$ & $\begin{array}{l}\text { CSV-Datei, } \\
\text { Diagramme als PDF oder JPG }\end{array}$ & $\begin{array}{l}\text { PNG-Bilddatei, CSV- } \\
\text { Datei, } \\
\text { Area Profiles } \\
\text { Datendownload } \\
\text { über Fingertips API } \\
\text { (Python package, } \\
\text { R package) } \\
\text { Download von } \\
\text { "analytical tools" }\end{array}$ & $\begin{array}{l}\text { Daten als CSV-Datei, } \\
\text { Karten und Diagram- } \\
\text { me als PNG-Datei, } \\
\text { „indicator summary" } \\
\text { als CSV-Datei } \\
\text { Download von „analy- } \\
\text { tical tools" }\end{array}$ & $\begin{array}{l}\text { Diagramme als Excel- } \\
\text { oder PowerPoint-Datei, } \\
\text { Tabellenwerte als CSV- } \\
\text { Datei }\end{array}$ \\
\hline \multicolumn{6}{|c|}{$\begin{array}{l}\text { API „Application Programming Interface", CSV "Comma-separated Values", ISD „Information Services Division”, NHS "National Health Service" } \\
\text { "Nachgewiesen im Internet Archive über Wayback Machine (04.11.2019); Kansas gehörte zu den Pilotstädten der City-Health-Dashboards, kann also auch } \\
\text { als Beispiel für diese gelten } \\
\text { "Mit einzelnen capacity-bezogenen Indikatoren oder Angaben, diese aber nicht als solche ausgewiesen oder gruppiert }\end{array}$} \\
\hline
\end{tabular}




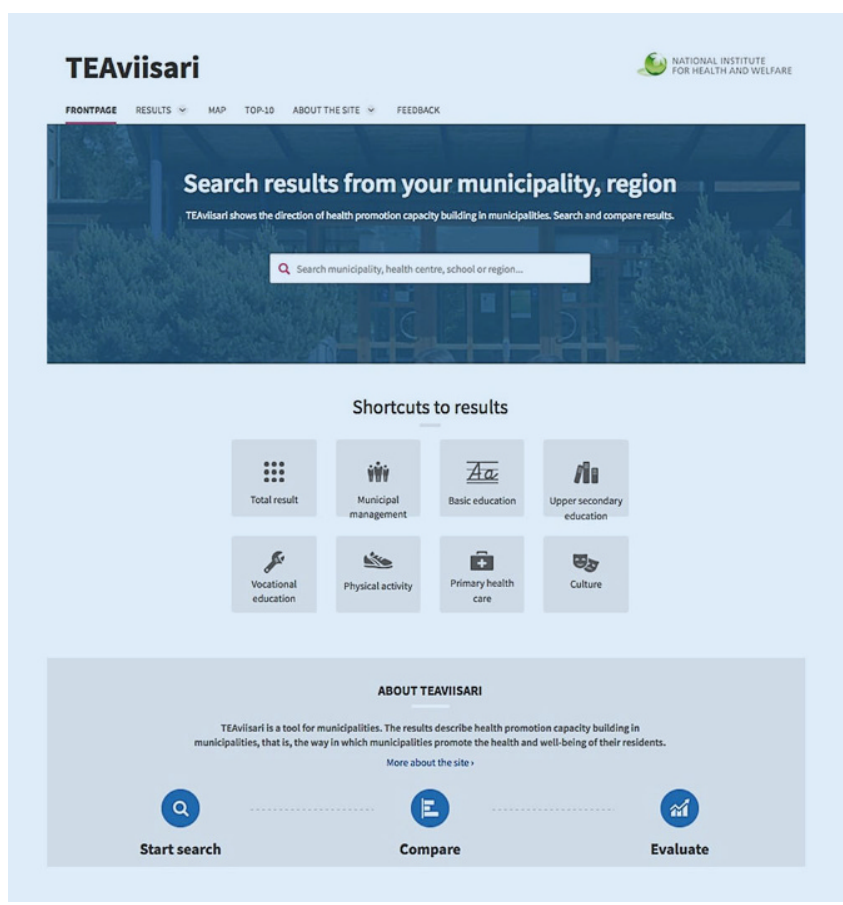

Abb. 1 ॥ Startseite des Benchmarkingtools "TEAviisari" (Bildschirmfoto; [51]) mit Suchfunktion und Quicklinks zur Gesamtsicht und den einzelnen "Sektoren"; in der oberen Menüzeile Navigationsmöglichkeiten zur Ergebnisansicht (• Abb. 2), Kartenansicht (• Abb. 3) und "Top-10“-Ansicht

[44]. Damit trägt DPH zu der bei Hawe et al. als dritte Stufe genannten Problemlösungsfähigkeit bei.

\section{TEAviisari - Finnland zeigt eine modellhafte Implementierung}

Eine von uns durchgeführte Webrecherche hat eine Reihe von interaktiven DPHTools und -Portalen zutage gefördert. Nachdem die klassische Recherche in Literaturinformationssystemen wenig erfolgreich gewesen war - nur in wenigen Fällen liegen wissenschaftliche Publikationen $\mathrm{zu}$ solchen Tools vor -, haben wir mit allgemeinen Suchmaschinen sowie Google Scholar nach entsprechenden Angeboten gesucht.

In die $\bullet$ Tab. 1 wurden solche aufgenommen, die möglichst viele der genannten Elemente aufweisen. Dabei waren uns vor allem die Aspekte der Datenintegration und interaktiven analytischen Aufbereitung wichtig. Dass außer TEAviisari keine weiteren dezidierten CB-Angebote gefunden werden konnten, ist einerseits bedauerlich, für unseren Zweck aber nicht entscheidend, da technische Funktionen für unterschiedliche Inhalte

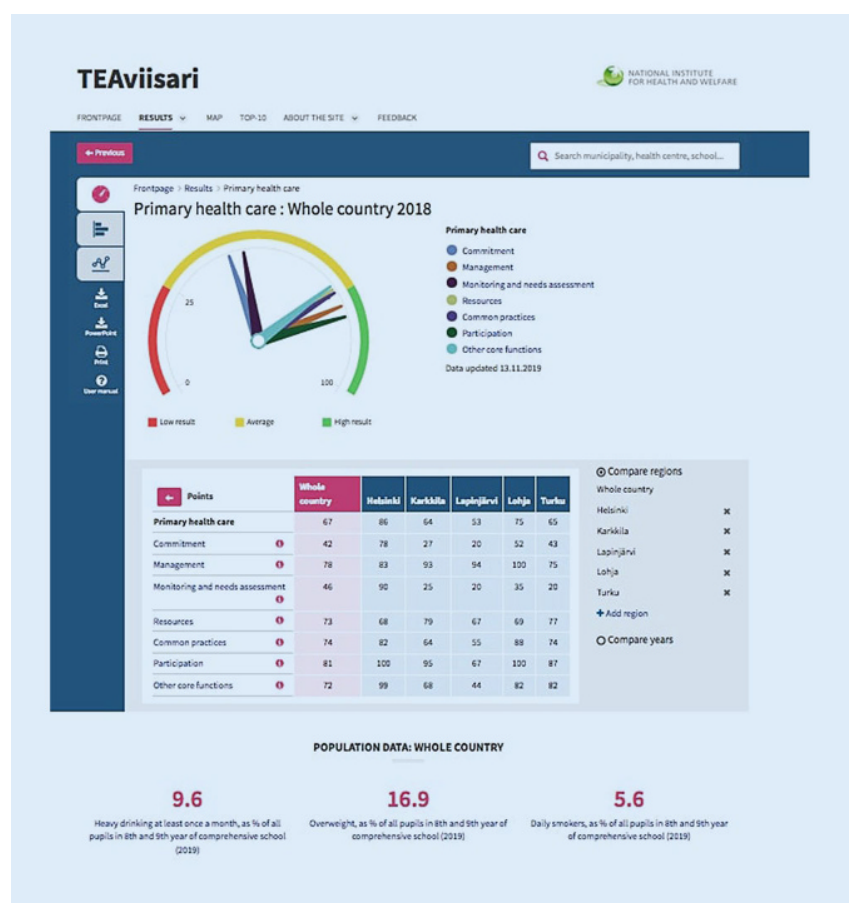

Abb. 2 ム Eine Ergebnisansicht im Benchmarkingtool „TEAviisari“ für „Primäre Gesundheitsversorgung" (Bildschirmfoto; [52]), hier mit individuell ausgewählter Vergleichsansicht für 5 Kommunen und landesweite Werte. In der "Gradmesser"-Darstellung sind die Zeiger auf der ersten Ebene gemäß den verschiedenen „Dimensionen“ farbcodiert; die Zeiger können für Verfeinerung angeklickt werden - bis auf die Ebene eines Einzelindikators. Standardeinstellung ist die Anzeige der neuesten Daten und der Vergleich mit landesweitem Wert. Durch Wechsel zu "compare years" kann der zeitliche Trend angezeigt werden. Die Optionen in der linken Menüspalte zeigen die Tabellenwerte als vergleichendes Balken- oder Liniendiagramm, die Grafiken können als PowerPoint-File heruntergeladen oder ausgedruckt werden. Die obere Navigationsleiste und die Suchfunktion laufen mit und erlauben den direkten Wechsel der Ansicht eingesetzt werden können und die konzeptionelle Nähe zu PH-Fragestellungen ja gegeben war. Die Inhalte der in die Tabelle aufgenommenen Tools umfassen meist gesundheitsbezogene Daten (z.B. Alkoholkonsum, Fast-Food-Restaurantdichte), auch Routinedaten der Versorgung und soziodemografische Informationen; Infrastruktur- und Kapazitätsdaten hingegen, die CB erfassen, spielen wenn überhaupt - eine untergeordnete Rolle (『Tab. 1). Eine Ausnahme bildet eben das finnische Tool TEAviisari, auf das wir uns deshalb im Folgenden konzentrieren.

TEAviisari setzt vieles von dem, was oben als Desiderat genannt wird, bereits um: Entwicklung eines erweiterbaren Public-Health-Indikatorensatzes, Nachnutzung von vorhandenen Daten im Sinne des Open-Data-Gedankens, trans- parente und zyklische Datenerhebung, strategische Ausrichtung im Rahmen eines Frameworks, innovative Datenvisualisierung, freie Bereitstellung, Zugang über ein interaktives Benutzerinterface mit vielfältigen Informationszugängen, Unterstützung von Vergleichs-, Benchmark- und Evaluationsprozessen, Unterstützung von sozialpolitischer Steuerung und Entscheidungsfindung, Vernetzung mit den Angeboten sotkanet. $\mathrm{fi}^{1}$ und Welfare Compass².

1 Datenbank und Informationsservice des National Institute for Health and Welfare (THL) mit frei verfügbaren Sozial- und Gesundheitsdaten - https://sotkanet.fi/sotkanet/en/index. Zugegriffen:02.11.2019.

${ }^{2}$ Der Welfare Compass bereitet ausgewählte Indikatoren in drei großen Profilbereichen auf -https://www.hyvinvointikompassi.fi/en/web/ hyvinvointikompassi.Zugegriffen:02.11.2019. 


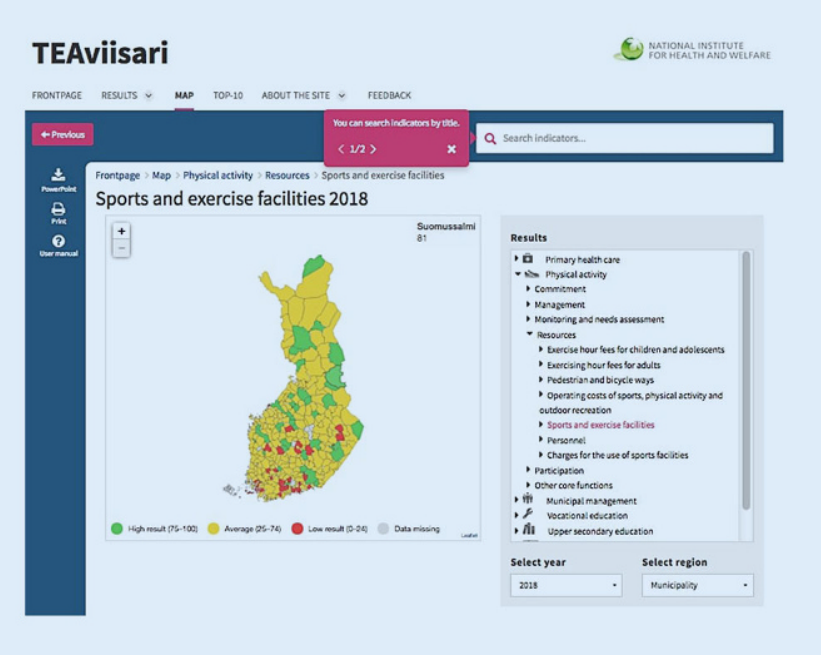

Abb. 3 A Eine Kartenansicht im Benchmarkingtool „TEAviisari“ (Bildschirmfoto; [53]). Ausgewählt die aus Einzelindikatoren aggregierte Sicht auf "sports und exercise facilities"; bei Mouse over erscheint in der oberen rechten Ecke der Wert der ausgewählten Einheit. Die Karte zeigt die Bewertung durch Ampelfarben an. Über die hierarchische Darstellung rechts können verschiedene Aggregationsebenen angesteuert werden. Ausgewählt werden können auch verschiedene Erhebungsjahre (hier:2018) und organisatorische Einheiten (hier: Municipality). Über sog. Breadcrumbs wird über der Resultatanzeige ein lineares „Zurück" angeboten. Die obere Navigationsleiste und die Suchfunktion laufen mit und erlauben den direkten Wechsel der Ansichten

TEAviisari $^{3}$ ist als digitale, webbasierte und frei zugängliche Plattform seit 2010 verfügbar, nach einem Relaunch im Jahr 2015 in überarbeiteter Form [45]. Die Benutzeroberfläche steht auf Finnisch, Schwedisch und Englisch zur Verfügung. Betreiber ist das National Institute for Health and Welfare (THL), das dem Ministry of Social Affairs and Health untersteht; Kooperationspartner sind das Ministry of Education and Culture sowie die Finnish National Agency for Education.

Der namensgebende Schlüsselbegriff ist „Health Promotion Capacity Building“ (HPCB) („terveydenedistämisaktiivisuus" [TEA]). Über die reine Datenrepräsentation hinaus ist die Leistung des Instruments die Umsetzung der Indikatoren in Dimensionen, Bereichsprofile

\footnotetext{
3 Alle Informationen zu TEAviisari stammen, sofern nicht anders vermerkt, von der englischsprachigen Fassung der Webseite (https:// teaviisari.fi/teaviisari/en/index?) oder den dort auf Englisch verfügbaren oder verlinkten Informationsmaterialien [Stand 02.11.2019], insbesondere Saaristo [46].
}

stände, zu den Erhebungsmethoden etc. geben Kilpeläinen et al. [48].

Für TEAviisari werden insgesamt 790 Einzelindikatoren erhoben, 771 davon sind auf $\mathrm{CB}$ bezogen; ein großer Anteil der Indikatoren (ca. 70\%) ist dichotom. Die Indikatoren werden zu verschiedenen Profilen aggregiert, können aber auch einzeln für einzelne - geografische, institutionelle - Einheiten aufgerufen werden.

Die Struktur von TEAviisari zeigt zum einen thematische Sektoren - Bereiche, für die Kapazitätsentwicklung gemessen werden soll. In der Praxis werden die für die Aktivitäten und Services verantwortlichen administrativen Einheiten betrachtet. Die Bereiche sind: Primary Health Care, Physical Activity, Municipal Management, Vocational Education, Upper Secondary Education, Basic Education, Culture. Die TEAviisari-„,Sektoren“ sind also das, was oben „Domäne“ genannt wurde. Diese Großbereiche sind über Quicklinks von der Startseite zu erreichen (• Abb. 1); innerhalb der Sektorendarstellung kann vertikal verfeinert werden auf einzelne Einheiten, die auch miteinander verglichen werden können, sowie auf Dimensionen, Subdimensionen, Indikatoren des HPCB-Frameworks (• Abb. 2).

Die zweite Achse bildet das HPCBFramework als Messinstrument mit 7 Dimensionen, die in Subdimensionen und Indikatoren aufgegliedert werden, die Dimensionen sind: Commitment, Management, Monitoring and Needs Assessment, Resources, Common Practices, Participation, Other Core Functions (-Abb. 2). Dieses Framework wird auf die "Sektoren“ angewendet; die offene Dimension „other core functions“ zeigt die Notwendigkeit sektorspezifischer Maßzahlen. Innerhalb der Ergebnisdarstellungen können verschiedene Ebenen angesteuert werden (- Abb. 2 und 3 ).

Das HPCB-Framework wird hinsichtlich Konsistenz und Effizienz kontinuierlich überprüft und weiterentwickelt, Ziel ist ein aussagekräftiges, aber möglichst schlankes Instrument [49].

Die individuelle Nutzbarkeit wird mitbestimmt durch die gezielte Suche nach geografischen oder verwaltungspolitischen Einheiten oder Institutionen 
verschiedener Ebenen (z.B. nach einer einzelnen Schule, einer Kommune, einer Region, einem Krankenhausbezirk). Dies ist ein Primäreinstieg in das Informationssystem, der mit dem Suchschlitz auf der Startseite angeboten ( $\bullet$ Abb. 1) und auf jeder Resultatedarstellung mitgeführt wird; damit kann von jeder Ansicht individuell auf die hinterlegten Einheiten verfeinert werden.

Mit Dimensionen, Sektoren und Einheiten entsteht ein dreidimensionales Modell der Kapazitätserschließung, -darstellung und -bewertung. Aus dieser Matrix werden verschiedene interaktive Ansichten generiert, die intuitiv zugängliche Visualisierungen nutzen wie die Qualitätsanzeige in Ampelfarben (- Abb. 2 und 3).

TEAviisari bietet dabei grundsätzlich 3 Standardansichten: (1) die Ergebnisansicht für Sektoren, Einheiten oder einzelne Institutionen, in deren Mittelpunkt eine kreisförmige „Gradmesser"-Visualisierung steht (• Abb. 2), (2) die Kartenansicht, die einen geografischen Überblick über die Sektoren mit Verfeinerungsfunktion gibt (• Abb. 3), hinzu kommt die „Top-10-Ansicht“, die kein Einheitenranking ist, sondern eine Einheitenansicht, die Stärken und Entwicklungsziele einer Kommune, Organisation oder andere Einheiten als positive oder negative Abweichung vom Landesdurchschnitt zeigt.

Das Qualitätsrating ergibt sich aus einer Umsetzung der erhobenen Daten in Punktwerte auf einer Skala von 0 bis 100. Jeder Indikator wird als Qualitätsmaß interpretiert; es werden Werte für die einzelnen Dimensionen und Subdimensionen berechnet, als Punktwert angegeben und mit Ampelfarben kategorisiert (0-24: rot - schlechte Qualität; 25-74: gelb - mittlere Qualität; 75-100: grün - gute Qualität).

Mit seinen vielfältigen Zugriffswegen, Einstiegen, Explorationspfaden und Kontexthilfen ermöglicht TEAviisari viele unterschiedliche Nutzungsszenarien. Das benutzerzentrierte Design des Interface ist sehr weit intuitiv verständlich. Dass dies gelingt, ist auf die konsequente Auslotung der Möglichkeiten von Datenrepräsentation, Datenverknüpfung und Datenvisualisierung zurückzuführen, die zu einem interaktiven Tool integriert wurden. Es geht also nicht um eine reine Verfügbarmachung von Datenreihen und ihren Diagrammen, sondern um das von Sedig und Ola avisierte „user-guided environment in which $\mathrm{PH}$ stakeholders can interact and reason with data" [44].

Genutzt werden die TEAviisari-Daten in verschiedenen Bereichen ${ }^{4}$ : u. a. für die Planung, das Management, die Verbreitung und die Evaluation von Gesundheitsförderungsaktivitäten, für regionale und landesweite Berichte, zur Vorbereitung von Gesetzen und der Evaluation ihrer Umsetzung, für die Evaluation schulischer Wohlfahrtsprogramme, zur Zielformulierung im Regierungshaushalt und -programm, für die Entwicklung von Strategien, politischen Handlungs- und Leitlinien sowie Programmen. Konkrete Beispiele hierfür sind die Bewertung schulischer Rauchpräventionsmaßnahmen (mit Daten aus TEAviisari und Schulgesundheitssurveys) oder ein Bericht auf der Basis der Daten zu Sport und körperlicher Aktivität. Auf der Webseite selbst finden sich „user stories“, die über die Nutzung des Tools Auskunft geben.

\section{Fazit}

Schon im Jahr 2000 sahen Ziglio et al. die erfolgreiche Weiterentwicklung des holistischen Ansatzes der Gesundheitsförderung u.a. in der Stärkung von lokalen Strukturen und Public-Health-Informationssystemen begründet [50]. Vielfältige Anstrengungen und weitere 2 Jahrzehnte später erscheinen uns diese Forderungen gültig und notwendig. Mit Blick auf Deutschland kommt man nicht umhin, neben der Heterogenität der Public-Health-Landschaft auch eine gewisse Verspätung hinsichtlich der Erhebung und Nutzung von Public-Health-Daten und des Einsatzes digitaler Technologien zur Analyse, Visualisierung und Dissemination solcher Informationen festzustellen.

\footnotetext{
${ }^{4}$ Informationen hierzu verdanken wir der persönlichen Auskunft von Timo Ståhl, Chief Specialist, PhD, Adjunct Professor National Institute for Health and Welfare.
}

DPH kann in dieser Situation $\mathrm{He}$ belwirkung entfalten und u.a. bereits erfasste Daten, die nicht genuin digital sein müssen, computergestützt zusammenführen, integrieren und in analytischen Visualisierungen aufbereiten. Dies befördert eine zielführende, intersektorale und datenbasierte Steuerung von Maßnahmen der Prävention und Gesundheitsförderung vor Ort und kann damit auch Entscheidungsprozesse unterstützen. Dass dies bereichs- und anwendungsspezifisch erfolgreich umgesetzt werden kann, zeigt die zunehmende Zahl solcher Angebote. TEAviisari ist eine für die öffentliche Hand modellhafte DPH-Implementierung, aber keine singuläre.

Wie jedoch unter den besonderen nationalen Verhältnissen von Datenverfügbarkeit und Zuständigkeiten das sektorenübergreifende Verständnis von CB in einer digitalen Infrastruktur fassbar sein könnte, bedarfzunächst der Bestandsaufnahme: Welche Daten liegen bereits vor und wie können sie verfügbar gemacht werden? Gibt es in Deutschland gut ausgebaute Domänen (wie die „Frühen Hilfen"), die beispielhaft und rasch vorangehen können? Zudem sollte eine empirische Erprobung im Kleinen erfolgen. Mit solchen Projekten könnte auch eine Verständigung über inhaltlich-konzeptionelle und technische Fragen erreicht werden: Welche „Domänen“, welche „Kapazitäten“ sollen abgebildet werden? Welche Teilmenge davon sollte ein Kernindikatorensatz $\mathrm{CB}$ enthalten? Wie muss ein Metadatenschema aussehen, um Datenintegration und -verfügbarkeit im oben ausgeführten Sinne praktisch zu unterstützen? Insgesamt kann das wohl nur in einem iterativen Prozess gelingen.

Dieser Artikel zeigt, dass auf internationalen Vorarbeiten zu Konzeptualisierung und technischer Umsetzung von CB-Monitoring aufgesetzt werden könnte. Wenn für Deutschland eine zentrale gesundheitspolitische Herausforderung weiterhin darin besteht, CB auf kommunaler Ebene zu etablieren, können durchdachte und benutzerorientierte digitale Informationssysteme hierbei einen wesentlichen Beitrag leisten. Die EURatspräsidentschaft Deutschlands 2020, die sich u.a. den Themen Digitalisierung 
und gemeinsamer Gesundheitsdatenraum widmet, bietet die Gelegenheit, die Entwicklung dieses Felds entscheidend voranzubringen.

\section{Korrespondenzadresse}

\section{Prof. Dr. Freia De Bock}

Bundeszentrale für gesundheitliche Aufklärung Maarweg 149-161,50825 Köln, Deutschland freia.debock@bzga.de

Danksagung. Wir danken Timo Ståhl, Chief Specialist, PhD, Adjunct Professor National Institute for Health and Welfare, und Christina Plantz, Bundeszentrale für gesundheitliche Aufklärung (BZgA), für wertvolle Hinweise bei der Erstellung des Manuskripts. Wir danken unseren anonymen Gutachter*innen für ihre hilfreichen und kritischen Kommentare, die beigetragen haben, den Text zu ergänzen und die Argumentation zu schärfen.

\section{Einhaltung ethischer Richtlinien}

Interessenkonflikt. M. Zens, Y. Shajanian Zarneh, J. Dolle und F. De Bock geben an, dass kein Interessenkonflikt besteht.

Für diesen Beitrag wurden von den Autoren keine Studien an Menschen oder Tieren durchgeführt. Für die aufgeführten Studien gelten die jeweils dort angegebenen ethischen Richtlinien.

Open Access. Dieser Artikel wird unter der Creative Commons Namensnennung 4.0 International Lizenz veröffentlicht, welche die Nutzung, Vervielfältigung, Bearbeitung, Verbreitung und Wiedergabe in jeglichem Medium und Format erlaubt, sofern Sie den/die ursprünglichen Autor(en) und die Quelle ordnungsgemäß nennen, einen Link zur Creative Commons Lizenz beifügen und angeben, ob Änderungen vorgenommen wurden.

Die in diesem Artikel enthaltenen Bilder und sonstiges Drittmaterial unterliegen ebenfalls der genannten Creative Commons Lizenz, sofern sich aus der Abbildungslegende nichts anderes ergibt. Sofern das betreffende Material nicht unter der genannten Creative Commons Lizenz steht und die betreffende Handlung nicht nach gesetzlichen Vorschriften erlaubt ist, ist für die oben aufgeführten Weiterverwendungen des Materials die Einwilligung des jeweiligen Rechteinhabers einzuholen.

Weitere Details zur Lizenz entnehmen Sie bitte der Lizenzinformation auf http://creativecommons.org/ licenses/by/4.0/deed.de.

\section{Literatur}

1. Zeeb H, Pigeot I, Schüz B (2020) Digital Public Health - ein Überblick. Bundesgesundheitsbl 63:137-144. https://doi.org/10.1007/s00103019-03078-7

2. Bär G (2015) Gesundheitsförderung lokal verorten. RäumlicheDimensionen und zeitliche Verläufe des
WHO-Setting-Ansatzes im Quartier. Springer VS, Wiesbaden

3. Boschek HJ, Kügler KJ (2001) Kommunale Gesundheitspolitik als Forschungsfeld von Public Health und Sozialwissenschaften. Gesundheitswesen 63:63-67.https://doi.org/10.1055/s-2001-12118

4. Walter U, Röding D, Kruse S, Quilling E (2018) Modelle und Evidenzen der intersektoralen Kooperation in der lebensweltbezogenen Prävention und Gesundheitsförderung. Ergebnisbericht.GKVSpitzenverband, Berlin

5. Volkenand K, Kruckenberg B, Schauermann L et al (2015) Daseinsvorsorge $=$ Gesundheitsförderung? Die Kommune als Dachsetting der Gesundheitsförderung: Was kann, was muss, was sollte? Gesundheitswesen 77:A262. https://doi.org/10. 1055/s-0035-156321

6. Altgeld T (2006) Mehr Prävention und Gesundheitsförderung kann nur vor Ort realisiert werden. Präv Gesundheitsf 1:24-32. https://doi.org/10. 1007/s11553-005-0011-z

7. Tsouros AD (2015) Twenty-seven years of the WHO European Healthy Cities movement: a sustainable movement for change and innovation at the local level. Health Promot Int 30:i3-i7. https://doi.org/ 10.1093/heapro/dav046

8. Nationale Präventionskonferenz (2019) Erster Präventionsbericht nach § 20d Abs. 4 SGB V. NPK, Berlin

9. Walter U, Nöcker G, Pawils S et al (2015) Memorandum - Prävention und Gesundheitsförderung nachhaltig stärken: Herausforderungen auf Bundes-, Landes- und kommunaler Ebene. Gesundheitswesen 77:382-388

10. Quilling E, KruseS (2018) Evidenzlage kommunaler Strategien der Prävention und Gesundheitsförderung: Eine Literatur- und Datenbankrecherche. Ergebnisbericht. GKV-Spitzenverband, Berlin

11. Geene R, Gerhardus A, Grossmann B et al (2019) Health in All Policies - Entwicklungen, Schwerpunkte und Umsetzungsstrategien für Deutschland. Zukunftsforum Public Health, Berlin

12. Süß W (2013) Lenzgesund - Vom Leuchtturmprojekt in die Perspektivlosigkeit? Gesundheitswesen. https://doi.org/10.1055/s-0033-1354062

13. Smith BJ, Tang KC, Nutbeam D (2006) WHO health promotion glossary: new terms. Health Promot Int 21:340-345. https://doi.org/10.1093/heapro/ dal033

14. Hawe P, King L, Noort M et al (2000) Indicators to help with capacity building in health promotion. NSW, Sydney

15. Potter C, Brough R (2004) Systemic capacity building: a hierarchy of needs. Health Policy Plan 19:336-345.https://doi.org/10.1093/heapol/ czh038

16. Labonte R, Laverack G (2001) Capacity building in health promotion. Part 1: For whom? And for what purpose? Crit Public Health 11:111-127. https:// doi.org/10.1080/09581590110039838

17. Aluttis C, Van den Broucke S, Chiotan C et al (2014) Public health and health promotion capacity at national and regional level: a review of conceptual frameworks. J Public Health Res. https://doi.org/ 10.4081/jphr.2014.199

18. Labonte R, Laverack G (2001) Capacity building in health promotion. Part 2: Whose use? And with what measurement? Crit Public Health 11:129-138. https://doi.org/10.1080/ 09581590110039847

19. Jackson SF, Perkins F, Khandor E et al (2006) Capacity building: integrated health promotion strategies: a contribution to tackling current and future health challenges. Health Promot Int 21:75-83. https://doi.org/10.1093/heapro/dal054

20. Frieden TR (2010) A framework for public health action: the health impact pyramid. Am J Public Health 100:590-595. https://doi.org/10.2105/ AJPH.2009.185652

21. Ståhl T (2018) Health in all policies: from rhetoric to implementation and evaluation-the Finnish experience. Scand J Public Health 46:38-46. https://doi.org/10.1177/1403494817743895

22. Brownson RC, Fielding JE, Green LW (2018) Building capacity for evidence-based public health: reconciling the pulls of practice and the push of research. Annu Rev Public Health 39:27-53. https://doi.org/10.1146/annurev-publhealth040617-014746

23. Ebbesen LS (2004) Issues in measuring health promotion capacity in Canada: a multi-province perspective. Health Promot Int 19:85-94. https:// doi.org/10.1093/heapro/dag408

24. Mittelmark MB, Fosse $E$, Jones $C$ et al (2005) Mapping European capacity to engage in health promotion at the national level: HP-Source.net. Promot Educ 12(Suppl 1):33-39. https://doi.org/ 10.1177/10253823050120010109x

25. Mittelmark MB, Wise $M$, Nam EW et al (2006) Mapping national capacity to engage in health promotion: overview of issues and approaches. Health Promot Int 21:91-98. https://doi.org/10. 1093/heapro/dal056

26. Burgun A, Bernal-Delgado $E$, Kuchinke $W$ et al (2017) Health data for public health: towards new ways of combining data sources to support research efforts in Europe. Yearb Med Inform 26:235-240. https://doi.org/10.15265/IY-2017034

27. Stockmann S, Kuhn J, Zirngibl A, Mansmann U (2008) Kommunale Gesundheitsberichterstattung in Deutschland: eine empirische Erhebung. Gesundheitswesen 70:679-683. https://doi.org/ 10.1055/s-0028-1100402

28. Harbers $M$, van der Wilk EA, Kramers PGN et al (2008) Dare to compare! Benchmarking Dutch health with the European Community Health Indicators (ECHI). RIVM report number 270051011. Bohn Stafleu Van Loghum, Houten

29. OECD (2015) Data-driven innovation: Big Data for growth and well-being. OECD, Paris

30. Dixon BE, Kharrazi H, Lehmann HP (2015) Public health and epidemiology informatics: recent research and trends in the United States. Yearb Med Inform 24:199-206. https://doi.org/10.15265/IY2015-012

31. Khoury MJ, lademarco MF, Riley WT (2016) Precision public health for the era of precision medicine. Am J Prev Med 50:398-401. https://doi. org/10.1016/j.amepre.2015.08.031

32. Hohwü L, Lyshol H, Gissler M et al (2013) Webbased versus traditional paper questionnaires: a mixed-mode survey with a nordic perspective. J Med Internet Res 15:e173. https://doi.org/10. 2196/jmir.2595

33. Croner CM (2003) Public health, GIS, and the Internet. Annu Rev Public Health 24:57-82. https:// doi.org/10.1146/annurev.publhealth.24.012902. 1408

34. Nykiforuk C, Flaman LM (2011) Geographic information systems (GIS) for health promotion and public health: a review. Health Promot Pract 12:63-73

35. Wilkinson MD, Dumontier M, Aalbersberg IJ et al (2016) The FAIR guiding principles for scientific data management and stewardship. Sci Data 3:160018. https://doi.org/10.1038/sdata.2016.18 


\section{Leitthema}

36. Scholz R, Bartelsman E, Diefenbach S et al (2018) Unintended side effects of the digital transition: European scientists' messages from a propositionbased expert round table. Sustainability 10:2001. https://doi.org/10.3390/su10062001

37. Carinci F (2015) Essential levels of health information in Europe: an action plan for a coherent and sustainable infrastructure. Health Policy 119:530-538 https://doi.org/10.1016/j.healthpol.2014.11.016

38. Ward M, Grinstein GG, Keim D (2015) Interactive data visualization: foundations, techniques, and applications, 2. Aufl. CRC Press, Boca Raton, London, New York

39. Ruppert T (2018) Visual analytics to support evidence-based decision making. Dissertation, Technische Universität Darmstadt

40. Gamache R, Kharrazi H, Weiner J (2018) Public and population health informatics: the bridging of Big Data to benefit communities. Yearb Med Inform 27:199-206. https://doi.org/10.1055/s0038-1667081

41. ZakkarM,Sedig K(2017)Interactivevisualization of public health indicators to support policymaking: an exploratory study. Online JPublic Health Inform. https://doi.org/10.5210/ojphi.v9i2.8000

42. Manorat R, Becker L, Flory A (2019) Global data visualization tools to empower decision-making in nutrition. Sight Life Mag 33:108-114

43. Sedig K, Parsons P, Dittmer M, Ola O (2012) Beyond information access: support for complex cognitive activities in public health informatics tools. Online J Public Health Inform. https://doi.org/10.5210/ ojphi.v4i3.4270

44. Sedig K, Ola O (2014) The challenge of Big Data in public health: an opportunity for visual analytics. Online J Public Health Inform. https://doi.org/10. 5210/ojphi.v5i3.4933

45. Saaristo V (2016) TEAviisari, a tool for benchmarking health promotion capacity building in Finland. http://eurohealthnet-magazine. eu/teaviisari-a-tool-for-benchmarking-healthpromotion-capacity-building-in-finland/. Zugegriffen: 02.11.2019

46. Puska P, Ståhl T (2010) Health in all policies - the Finnish initiative: background, principles, and current issues. Annu Rev Public Health 31:315-328. https://doi.org/10.1146/annurev. publhealth.012809.103658

47. Kilpeläinen K, Tuomi-Nikula A, Thelen J et al (2012) Health indicators in Europe: availability and data needs. Eur J Public Health 22:716-721. https://doi. org/10.1093/eurpub/ckr195

48. Kilpeläinen K, Parikka S, Koponen P et al (2016) Finnish experiences of health monitoring: local regional, and national data sources for policy evaluation. Glob Health Action 9:28824. https:// doi.org/10.3402/gha.v9.28824

49. Saaristo V, Hakamäki P, Koskinen $\mathrm{H}$ et al (2018) The comparative and objective measurement of health promotion capacity-building: from conceptual framework to operationalization. Glob Health Promot. https://doi.org/10.1177/ 1757975918769608

50. Ziglio E, Hagard S, Griffiths J (2000) Health promotion development in Europe: achievements and challenges. Health Promot Int 15:143-154. https://doi.org/10.1093/heapro/15.2.143

51. TEAviisari (2019) Startseite. https://teaviisari.fi/ teaviisari/en/index.Zugegriffen:29.11.2019

52. TEAviisari (2019) Ergebnisansicht. https:// teaviisari.fi/teaviisari/en/tulokset. Zugegriffen: 29.11.2019

53. TEAviisari (2019) Kartenansicht. https://teaviisari. fi/teaviisari/en/kartta.Zugegriffen:29.11.2019 